\title{
A comparative study of sound localization acuity of congenital blind and sighted people
}

\author{
Makoto Ohuchi ${ }^{1,2, *}$, Yukio Iwaya ${ }^{1,3}$, Yôiti Suzuki ${ }^{1,3}$ and Tetsuya Munekata ${ }^{4}$ \\ ${ }^{1}$ Graduate School of Information Sciences, Tohoku University \\ ${ }^{2}$ Department of Information Science for Human Welfare, Tohoku Fukushi University \\ ${ }^{3}$ Research Institute of Electrical Communication, Tohoku University \\ ${ }^{4}$ National Institute of Special Education
}

( Received 12 December 2005, Accepted for publication 24 May 2006)

Keywords: Sound localization, Distance perception, Visual impairments, Auditory scanning PACS number: 43.66.Qp [doi:10.1250/ast.27.290]

\section{Introduction}

For the visually impaired, information provided by sound is particularly important in recognizing their surroundings since they have difficulty in using visual information. That is, they mainly perceive attributes of various objects and spatial information around them utilizing sound. Therefore, the sound localization acuity of visually impaired people is estimated to be better than that of sighted people. Several studies of this topic have shown that the sound localization acuity of blind adults is higher than that of sighted adults and adults with lateonset blindness [1-3]. In some of these studies, only specific directions, such as in front of the subject and lateral to the subject, were measured. It is essential, however, to investigate such acuity for all azimuthal angles. Kellogg [3] reported that blind subjects often rotate their heads when they are searching for obstacles. He called this behavior "auditory scanning." This strongly suggests that the use of dynamic cues of sound inputted to two ears caused by head rotation is crucial for realizing precise sound localization.

Therefore, in this study, directional and distance perceptions as related to horizontal plain localization and distance at various azimuthal angles were investigated in terms of sound localization acuity. Moreover, the authors examined the difference in sound localization acuity between sighted and blind adults, and between the case in which the rotation of a listener's head was allowed and that in which the head was fixed.

\section{Measurement of Sound Localization acuity}

\subsection{Listeners}

The listeners were four congenital blind adults and four sighted adults. All listeners were males with normal hearing. 2.2. Equipment and procedure

Measurement was conducted in a soundproof room. Twelve loudspeakers were arranged at 30 degrees intervals in a circle with a radius of $1.5 \mathrm{~m}$. The listener was instructed to sit in the circle so that the center of the axis of his ears coincided with the center of the circle.

The height of the loudspeakers was set at the level of the listener's ears. A sound stimulus was randomly presented from one of the 12 loudspeakers. The sound stimulus was low-pass-filtered pink noise $\left(f_{\mathrm{c}}=16 \mathrm{kHz}\right)$, with a $2 \mathrm{~s}$ dura-

\footnotetext{
*e-mail: makoto@tfu-mail.tfu.ac.jp
}

tion. Sound pressure level was set at $70 \mathrm{~dB}$ at the center of the circle without a listener. The listener was asked to report both the azimuth of the perceived sound location using the hour hand of a clock and the distance [cm] from the center position of the head to the perceived sound image. Listeners were asked to report both the perceived azimuth and perceived distance $[\mathrm{cm}]$ of the sound image from the center position. As a result of a discussion with blind participants, the perceived azimuth was reported as the time based on the hour hand of a clock. For example, zero, two and six o'clock respectively mean 0, 45, and 180 degrees. The sighted participants also reported the azimuth by this method. The resolution of the reported time was not specified to the participants. However, all the reported times were expressed as 00 minutes or 30 minutes. This means that each perceived azimuth was reported with a resolution of 15 degrees. After that, the listener also reported the distance by subjective estimation. The sighted listeners were blindfolded before they came into the soundproof room to prevent them from knowing the position of the loudspeakers, this procedure being important for equalizing conditions for the sighted and blind listeners in localizing sound stimuli.

This experiment was conducted under the following two conditions.

- condition with listener's head fixed (hereafter, CHF)

- condition with listener's head free to rotate (hereafter, CHR)

In one trial, a stimulus was presented from one of the 12 loudspeakers and the listener evaluated the azimuthal direction and the distance to the loudspeaker. One session consisted of 12 trials. Under each of the two conditions, $\mathrm{CHF}$ and CHR, five sessions were conducted for each listener. For CHR, each sound stimulus was presented after visually checking that the listener's face had turned to the front.

2.3. Results

2.3.1. Sound localization error

Figure 2 shows the sound localization errors for the following four conditions: Blind/CHF, Blind/CHR, Sighted/ $\mathrm{CHF}$, and Sighted/CHR. Sound localization error was calculated as the average of the differences between the azimuth of a sound source and the azimuth of the sound localization perceived by all the listeners. The abscissa expresses the four conditions of the experiment, and the 


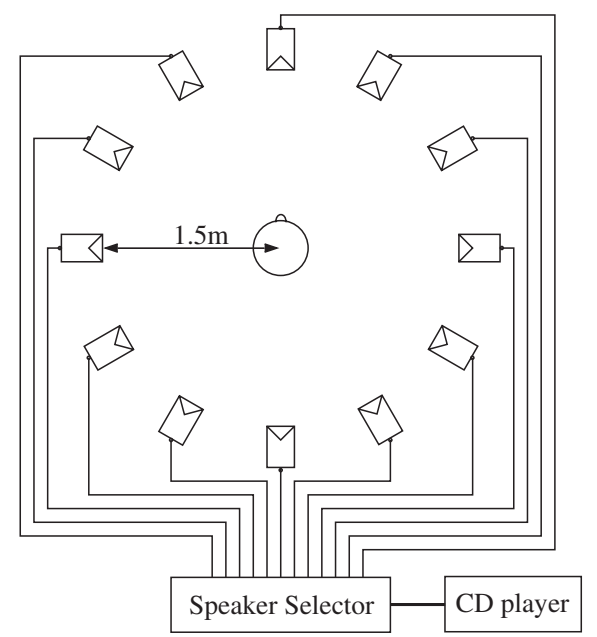

Fig. 1 Equipment for measuring sound localization acuity.

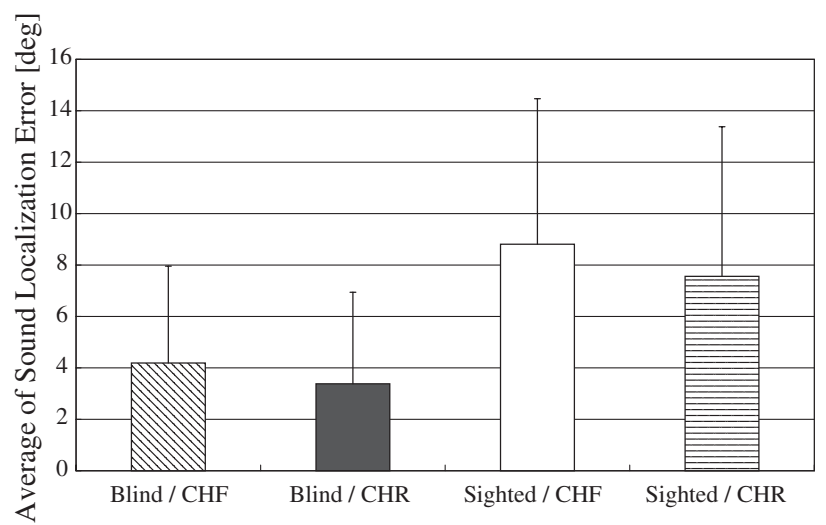

Fig. 2 Comparison of sound localization error.

ordinate expresses sound localization error in degrees. Error bars show standard deviations. The figure shows that the sound localization errors of the blind listeners were lower than those of the sighted listeners under both CHR and CHF, and that the errors under $\mathrm{CHR}$ were slightly lower than those under CHF irrespective of whether or not the listeners were blind or sighted.

Figure 3 shows the sound localization errors at every azimuth of the sound sources for the four conditions. The abscissa expresses the clockwise azimuth of the sound sources presented, and the ordinate expresses sound localization error in degrees. Error bars show standard deviations. The figure shows the following points.

(1) In most azimuths of the sound sources, the sound localization errors of the blind listeners were lower than those of the sighted listeners.

(2) In most azimuths of the sound sources, the sound localization errors of the blind listeners were less than 7 degrees at most.

(3) For azimuths of 0 and 180 degrees, there were hardly any sound localization errors of the blind listeners.

(4) The localization errors for the semicircle on the right (30 to 150 degrees) and those for the semicircle on the left (210 to 330 degrees) were not symmetrical.

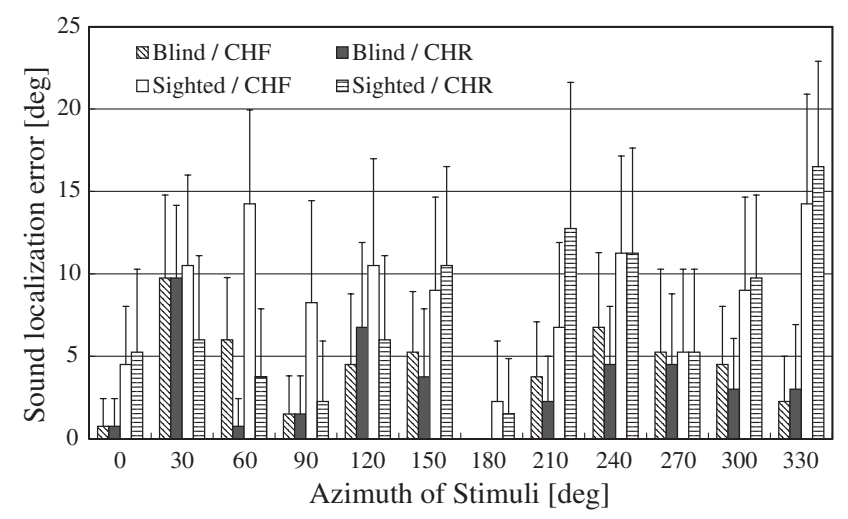

Fig. 3 Comparison of sound localization error at every azimuth.

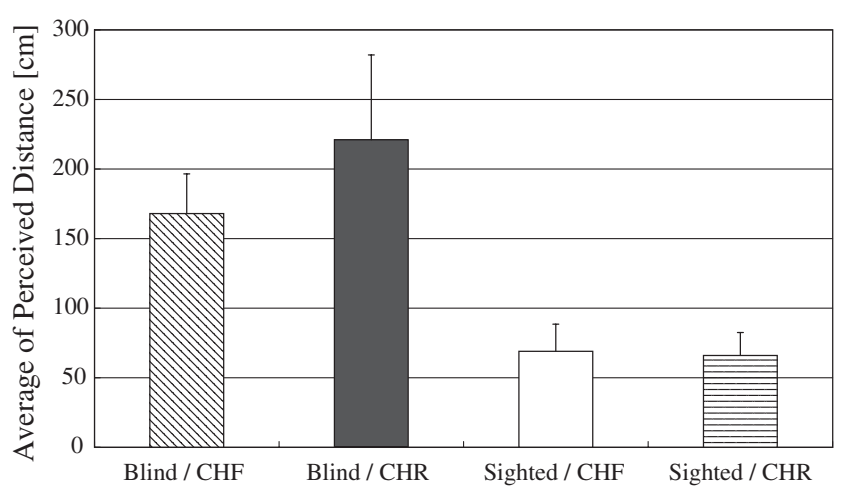

Fig. 4 Comparison of perceived distance.

A three-way analysis of variance (ANOVA) was conducted using three factors: VISION (blind or sighted), HEAD (CHF or CHR), and AZIMUTH (0 to 330 degrees).

As a result, the main effect of VISION was statistically significant $(F(1,6)=8.28, p<0.05)$, but neither the effects of HEAD and AZIMUTH nor the interactions among the three factors were significant. Therefore, the result showed that the sound localization error of the blind listeners was smaller than that of sighted listeners, irrespective of the allowance of head rotation.

2.3.2. Distance perception

Figure 4 shows perceived distances. The abscissa expresses the four experimental conditions and the ordinate expresses the average perceived distance reported in centimeters. Error bars show standard deviations. When the differences between the perceived distance and the actual sound source distance were compared, for the blind listeners, the difference was only $+18 \mathrm{~cm}$ under $\mathrm{CHF}$, but no less than $+71 \mathrm{~cm}$ under CHR. In contrast, for the sighted listeners, the difference was $-81 \mathrm{~cm}$ under $\mathrm{CHF}$ and $-84 \mathrm{~cm}$ under $\mathrm{CHR}$. Therefore, for the blind listeners, under CHF, the perceived distance is quite exact, but for the sighted listeners, the result suggests that they tend to underestimate the distance to a sound source.

Figure 5 shows the perceived distance at every azimuth of the sound sources for all four conditions. Several facts can be seen from this figure. 


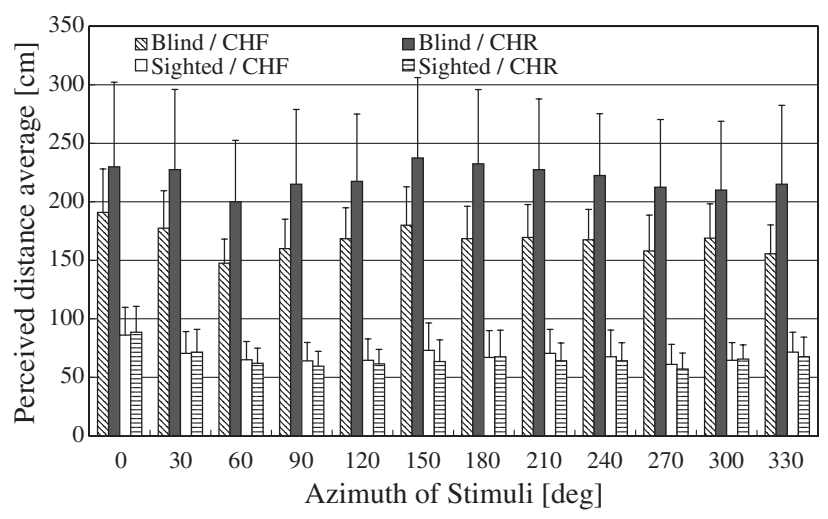

Fig. 5 Comparison of perceived distance at every azimuth.

(1) In all the azimuths of the sound sources, for the sighted listeners, there were few differences between CHF and CHR.

(2) Perceived distance was not dependent on the azimuth of the sound sources.

A three-way ANOVA was then conducted with three factors: VISION (blind or sighted), HEAD (CHF or CHR), and AZIMUTH (0 to 330 degrees).

As a result, the main effect of VISION conditions was statistically significant $(F(1,6)=6.67, p<0.05)$, but neither the effects of HEAD and AZIMUTH nor the interactions among the three factors were significant. That is, as regards the perceived distance, a significant difference is observed between blind and sighted people, irrespective of the allowance of head rotation.

\section{Discussion}

Irrespective of the allowance of head movement, the sound localization errors of the blind was lower than that of sighted listeners. This is in agreement with the findings of a comparative study of the sound localization acuity of blind and sighted listeners by Lessard [4]. Although the authors expected a reduction in sound localization error when head rotation was allowed [5-7], no significant difference was observed. Further investigation seems necessary to clarify the role and effect of head movement.

The authors observed an interesting action in all the blind listeners during the experiment, namely, during CHR, they intensively rotated their heads. It seemed that they were rotating the face to detect a sound source in front of it. In contrast, the sighted listeners only slightly rotated their heads to the right and left.

Table 1 shows the perceived distances for all listeners.

The average perceived distance under CHR for the blind listeners was more than $220 \mathrm{~cm}$. This is because the average of

Table 1 Perceived distances [cm].

\begin{tabular}{c|rccc|cccc}
\hline & \multicolumn{4}{|c|}{ Blind Listener } & \multicolumn{4}{c}{ Sighted Listener } \\
\hline Listener & A & B & C & D & E & F & G & H \\
CHF & 99 & 208 & 215 & 149 & 59 & 30 & 62 & 124 \\
CHR & 100 & 208 & 413 & 162 & 57 & 34 & 60 & 113 \\
\hline
\end{tabular}

listener $\mathrm{C}$ was $413 \mathrm{~cm}$. The average perceived distances for the other three blind listeners were $152 \mathrm{~cm}$ under $\mathrm{CHF}$ and $157 \mathrm{~cm}$ under CHR. Namely, in this experiment, the number of blind listeners showing smaller errors than the average error in distance perception was more than that of sighted listeners. This suggests that blind listeners would have better acuity in distance perception than sighted listeners, though the difference is not statistically significant since the number of listeners in our experiment was limited. This seems an interesting issue to clarify in future studies. Incidentally, the $\mathrm{CHF}$ average for listener $\mathrm{C}$ was $215 \mathrm{~cm}$. The influence of head movement appeared only in distance perception, not in sound localization. Although it is not clear from the results of this experiment, distance perception might be misguided by an intentional movement of the head for two seconds. This should be clarified in an additional experiment. The perceived distance of a sound source tends to be shorter than the actual distance [8,9]. In this study, only the results for the sighted listeners agree well with this tendency. For the blind, in contrast, the perceived distance corresponded accurately to the actual sound source distance. This also suggests that the blind listeners had high acuity for distance perception.

\section{Conclusion}

The authors measured the sound localization acuity of blind and sighted adults in a horizontal plane, and compared the acuity of the blind with that of the sighted. Results showed that the acuity of the blind is significantly superior to that of the sighted for both sound localization and distance perception at any azimuth of the horizontal plane.

\section{Acknowledgments}

This research was partially supported by the Ministry of Education, Culture, Sports, Science and Technology Grantin-Aid for Exploratory Research, 14658054, 2002-2004 and by Consortium R\&D Projects for Regional Revitalization of the Ministry of Economy Trade and Industry of Japanese Government, 2003-2004.

\section{References}

[1] C. E. Rice, "Early blindness, early experience and perceptual enhancement," Res. Bull. Am. Found. Blind, 22, 1-22 (1970).

[2] J. Juurmaa, "On the accuracy of obstacle detection by the blind Part 1," New Outlook Blind, 64(3), pp. 65-72 (1970).

[3] W. N. Kellogg, "Sonar system of the blind," Science, 137, 399-404 (1962).

[4] N. Lessard, M. Pare, F. Lepore and M. Lassonde, "Early-blind human subjects localize sound sources better than sighted subjects," Nature, 395, 278-280 (1998).

[5] J. Kawaura, Y. Suzuki, F. Asano and T. Sone, "Sound localization in headphone reproduction by simulating transfer functions from the sound to the external ear," J. Acoust. Soc. Jpn. (E), 12, 203-216 (1991).

[6] Y. Iwaya, Y. Suzuki and S. Takane, "Effects of listener's head movement on the accuracy of sound localization in virtual environment," Proc. 18th Int. Congr. Acoust. (ICA 2004), pp. 997-1000 (2004).

[7] Y. Iwaya, Y. Suzuki and D. Kimura, "Effects of head movement on front-back error in sound localization," Acoust. Sci. \& Tech., 24, 322-324 (2003). 
[8] G. von Békésy, "The moon illusion and similar auditory phenomena," Am. J. Psychol., 62, 540-552 (1949).

[9] P. Zahorik, D. S. Brungart and A. W. Bronkhorst, "Auditory distance perception in humans: A summary of past and present research," Acta Acustica, 91, 409-420 (2005). 